\title{
Development and Validation of an LC-MS/MS Method for Determination of Catalpol and Harpagide in Small Volume Rat Plasma: Application to a Pharmacokinetic Study
}

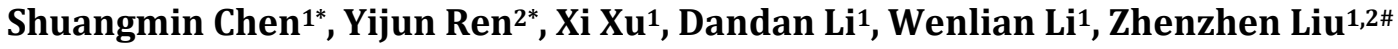 \\ ${ }^{1}$ Department of Medical Function, Health Medical Center, Yangtze University, Jingzhou, China \\ ${ }^{2}$ Department of Drug Clinical Trails, Center Hospital of Jingzhou, Jingzhou, China \\ Email: "liuzz_yangtze@hotmail.com
}

How to cite this paper: Chen, S.M., Ren, Y.J., Xu, X., Li, D.D., Li, W.L. and Liu, Z.Z. (2018) Development and Validation of an LC-MS/MS Method for Determination of Catalpol and Harpagide in Small Volume Rat Plasma: Application to a Pharmacokinetic Study. Yangtze Medicine, 2, 95-107. https://doi.org/10.4236/ym.2018.22011

Received: January 9, 2018

Accepted: June 8, 2018

Published: June 11, 2018

Copyright $\odot 2018$ by authors and Scientific Research Publishing Inc. This work is licensed under the Creative Commons Attribution International License (CC BY 4.0).

http://creativecommons.org/licenses/by/4.0/

\section{c) (i) Open Access}

\begin{abstract}
A rapid and sensitive liquid chromatography-tandem mass chromatography (LC-MS/MS) method has been developed and validated for simultaneous determination of catalpol and harpagide in rat plasma. The samples were extracted by one-step protein precipitation and separated on a SunFireTM $\mathrm{C}_{18}$ column $(100 \mathrm{~mm} \times 2.1 \mathrm{~mm}, 3.5 \mu \mathrm{m}$; Waters $)$ using acetonitrile- $10 \mathrm{mM}$ ammonium formate as mobile phase at a flow rate $0.3 \mathrm{~mL} / \mathrm{min}$ in gradient mode. The analytes were detected without interference in Multiple Reaction Monitoring (MRM) mode with negative electrospray ionization. Linear responses were obtained for catalpol ranging from 20 to $5000 \mathrm{ng} / \mathrm{mL}$ and harpagide ranging from 10 to $2500 \mathrm{ng} / \mathrm{mL}$. Coefficients of correlation (r) for the calibration curves were more than 0.99 for both analytes. Intra- and inter-day accuracy and precision were within the acceptable limits of less than $15.0 \%$ at all concentrations. The quantitation method was successfully applied for simultaneous estimation of catalpol and harpagide after oral administration of Zeng Ye Decoction.
\end{abstract}

\section{Keywords}

LC-MS/MS, Pharmacokinetics, Catalpol, Harpagide, Zeng Ye Decoction

\section{Introduction}

Zeng Ye Decoction (ZYD), a well-known Chinese herbal formula, is an aqueous extract of Radix Scrophulariae, Radix Rehmanniae and Radix Ophiopogonis at

${ }^{\star}$ The two authors contributed equally to this work.

\#Corresponding author. 
the weight ratio of 5:4:4. This prescription is widely used in clinical, and especially plays an important role in moisturizing dryness, promoting the production of body fluids, and primarily treating functional constipation associated with yin deficiency [1]. Catalpol and harpagide are two characteristic iridoid glycosides extensively investigated in Zeng Ye Decoction. Catalpol is now expanding rapidly and making its way to the forefront of anti-diabetic [2] [3] [4], anti-inflammatory [5] [6] [7] and neuroprotective research [8] [9] [10] [11]. Harpagide has also presented anti-inflammatory, anti-osteoporotic and antibacterial activities [12] [13] [14]. As the major bioactive components in ZYD, catalpol and harpagide are speculated to be the representative ingredients that contribute to the pharmacodynamics material basis. Due to the complexity of constituents in Chinese medicine, one or several compounds are generally selected to investigate the pharmacokinetic properties of the prescription and assess the compatibility of compound recipe which is significant in the evaluation of drug therapeutic effect, dose adjustment and the rational use of the drug in the clinic.

There are many analytical methods developed to determine catalpol [15] [16] [17] [18] and harpagide [19] [20] [21]. However, the method for simultaneous determination of catalpol and harpagide by LC-MS/MS has not been mentioned in the literatures. Based on these reasons, an LC-MS/MS method for simultaneous quantification of catalpol and harpagide by one-step protein precipitation was developed and validated in this study. This method is simple, sensitive and reproducible, and successfully applied to pharmacokinetic study of ZYD extract after oral administration to rats.

\section{Experimental}

\subsection{Reagents, Chemicals and Animals}

The reference standard of harpagide and catalpol (purity $>98.0 \%$, HPLC) were purchased from Beijing Solarbio Science \& Technology Co. Ltd (Beijing, China), and their structures are shown in Figure 1. Diclofenac sodium (purity $>99.5 \%$ ) used as the internal standard (IS), was obtained from Shanghai Yuanye Bio-Technology Co. Ltd (Shanghai, China). The crude drugs of Radix scrophulariae, Radix Ophiopogonis and Radix rehmannia were purchased from Tong Ren Tang Chinese Medicine Co., Ltd. (Beijing, China) and carefully authenticated by Dr. Liang Peng according to Chinese pharmacopoeia (The Pharmacopoeia Commission of PRC, 2015). Solvents used as eluents for liquid chromatography-mass spectrometry were HPLC grade from Fisher Scientific (FairLawn, NJ, USA). All the other reagents were analytical grade and redistilled water was used throughout this study.

Specific pathogen free (SPF) Wistar rats $(200-230 \mathrm{~g})$ were purchased from the Experimental Center of Hubei Medical Scientific Academy (SCXK2015-00018, Hubei, China). The animal experiments were performed in the Center for Animal Experiment of Hubei College of Chinese Medicine (Jingzhou, China), which has been accredited by the Association for Assessment and Accreditation of Laboratory Animal Care International. All animal experiment procedures were 


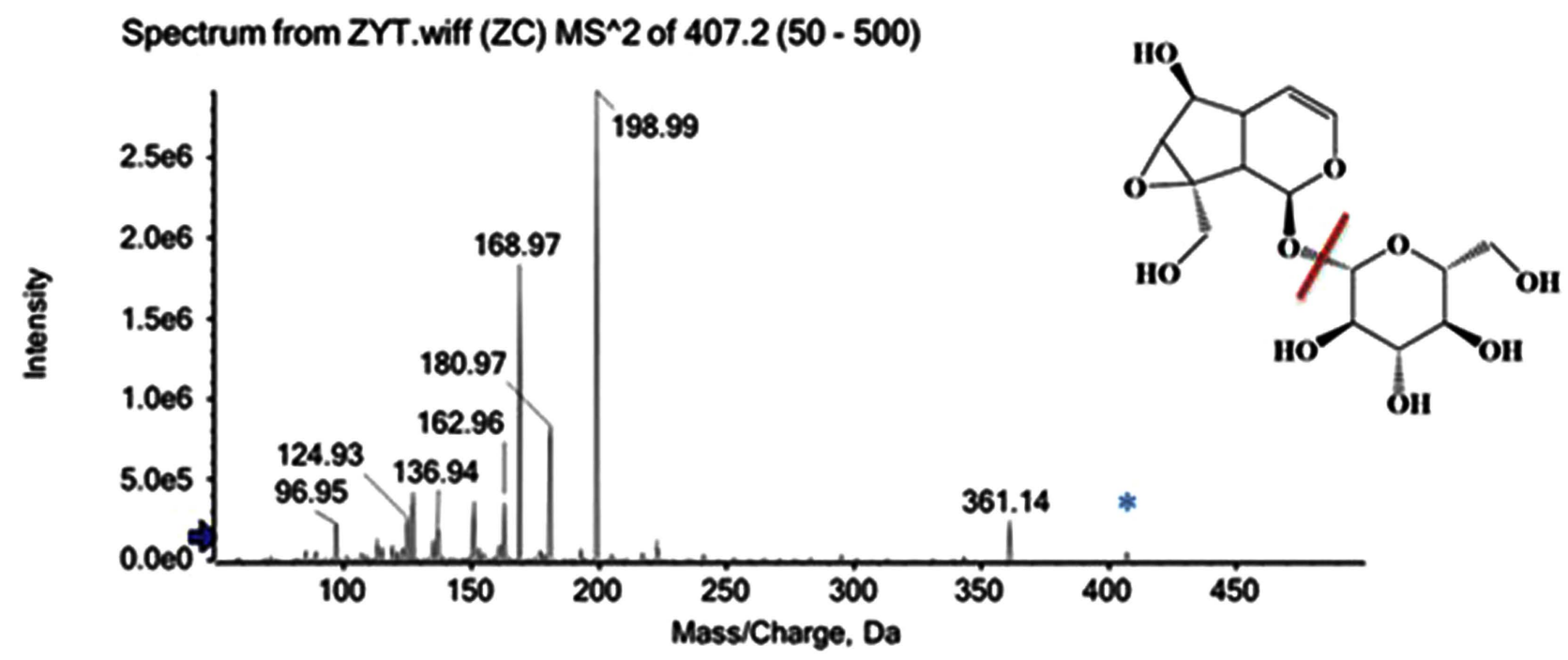

(a)

\section{Spectrum from ZYT.wiff (HBG) MS^2 of 409.2 (50 - 500)}

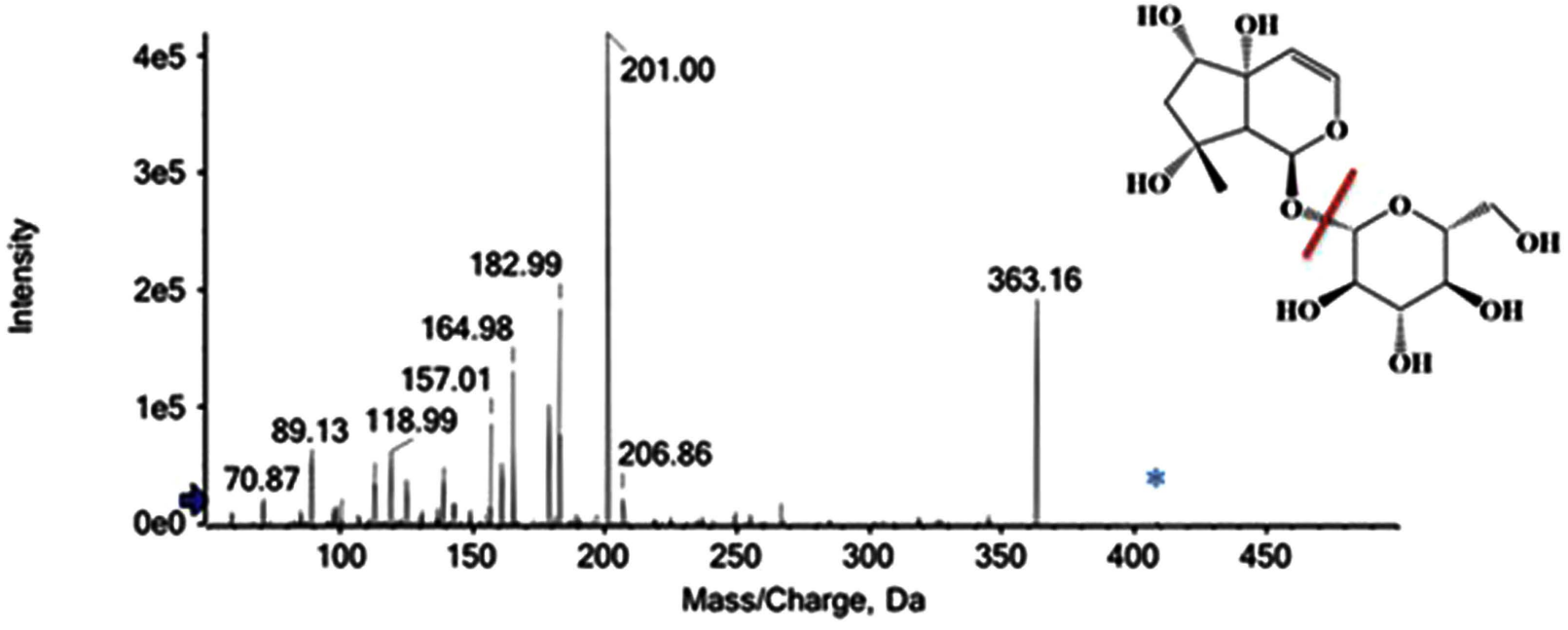

(b)

Figure 1. Chemical structures and MS-MS spectra of catalpol (a) and harpagide (b).

approved and performed in accordance with the Guidelines for the Care and Use of Laboratory Animals of the Chinese Animal Welfare Committee.

\subsection{Preparation of Aqueous Extract of ZYD}

ZYD was obtained by decocting the crude drugs of Radix Scrophulariae, Radix Ophiopogonis and Radix Rehmannia in the proportion of 5:4:4 according to the traditional use. In brief, the crude drugs (130 g) were extracted with 20 folds water for $1 \mathrm{~h}$ by reflux extraction. After filtered, the supernatant was collected and evaporated to dryness. Then the residue was reconstituted in water to obtain concentrations equivalent to $0.25 \mathrm{~g} / \mathrm{mL}$ of Radix Scrophulariae, $0.2 \mathrm{~g} / \mathrm{mL}$ of Radix Ophiopogonis and $0.2 \mathrm{~g} / \mathrm{mL}$ of Radix Rehmanniae. The extracts were kept at $4^{\circ} \mathrm{C}$ for further oral gavage to rats. To calculate the administered dose, the con- 
tent of catalpol and harpagide in ZYD were quantitatively analyzed. The solution obtained was diluted 50 times, filtered through a $0.22 \mathrm{~mm}$ membrane, and then quantitatively assayed by LC external standard method. The contents of catalpol and harpagide were determined to be 2.37 and $1.28 \mathrm{mg} / \mathrm{mL}$ in ZYD given to rats, respectively.

\subsection{Instruments and LC-MS Conditions}

Samples analyses were performed on a Shimadzu HPLC system (Shimadzu, Kyoto, Japan) interfaced with an API 4500 Qtrap (AB Sciex, Concord, Canada). The HPLC system consists of an LC-20ADXR binary pump, a SIL-20ACXR autosampler, a CTO-20AC column oven, a DGU-20A3 degasser and a CBM-20A controller. Chromatographic separation was performed on Waters SunFireTM $\mathrm{C}_{18}$ column (100 mm $\times 2.1 \mathrm{~mm}, 3.5 \mu \mathrm{m}$; Waters, Milford, MA, USA) maintained at $40^{\circ} \mathrm{C}$. The mobile phases consisted of acetonitrile as mobile phase $\mathrm{A}(\mathrm{A})$ and $10 \mathrm{mM}$ ammonium formate as mobile phase B (B). The flow rate was set at 0.3 $\mathrm{mL} / \mathrm{min}$. The gradient elution program optimized for separation efficiency was as follows: 0 - $2.0 \mathrm{~min}, 2 \% \mathrm{~A} ; 2.0$ - $3.0 \mathrm{~min}, 2 \%$ - 40\% A; 3.0 - $6.0 \mathrm{~min}, 40 \%-90 \%$ A; 6 - 7 min, $90 \% \mathrm{~A}$. The injection volume was $2.0 \mu \mathrm{L}$.

A 4500 QTRAP $^{\circledR}$ mass spectrometer (AB SCIEX, Concord, Canada) equipped with a Turboionspray ${ }^{\oplus}$ source (TIS) was operated in the negative ion mode with MRM (multiple reaction monitoring) for LC-QTRAP-MS/MS analysis. The MS parameters were optimized as follows: TIS temperature, $500^{\circ} \mathrm{C}$; ionspray voltage, $-4500 \mathrm{~V}$; curtain gas, nitrogen, 30 ; nebulizing gas, 50; TIS gas, 50; declustering potential, $-40 \mathrm{~V}$ for catalpol, $-40 \mathrm{~V}$ for harpagide and $-29 \mathrm{~V}$ for IS; entrance potential, $-10 \mathrm{~V}$; collision energy (CE), $-25 \mathrm{eV}$ for catalpol, $-27 \mathrm{eV}$ for harpagide and $-31 \mathrm{eV}$ for IS. The following MRM transitions were used: $\mathrm{m} / \mathrm{z} 407.2 \rightarrow$ 199.0 for catalpol, $409.2 \rightarrow 201.0$ for harpagide and $294.0 \rightarrow 214.0$ for IS, respectively.

\subsection{Stock Solution, Calibration Standards and Quality Control Samples}

Stock solutions of catalpol, harpagide and IS were prepared in methanol-water $(50: 50, \mathrm{v} / \mathrm{v})$ with a final concentration of $0.5 \mathrm{mg} / \mathrm{mL}, 0.25 \mathrm{mg} / \mathrm{mL}$ and 0.1 $\mathrm{mg} / \mathrm{mL}$, respectively. Working solutions were prepared by diluting respective stocking solution to suitable concentrations of $0.1-25 \mu \mathrm{g} / \mathrm{mL}$ for catalpol and $0.05-12.5 \mu \mathrm{g} / \mathrm{mL}$ for harpagide with methanol-water (50:50, v/v). Quality control (QC) working solutions at concentrations of $0.1,0.2,2.0$ and $20 \mu \mathrm{g} / \mathrm{mL}$ for catalpol and $0.05,0.1,1.0$ and $10 \mu \mathrm{g} / \mathrm{mL}$ for harpagide were made from a separately prepared $400 \mu \mathrm{g} / \mathrm{mL}$ stock solution of catalpol and $200 \mu \mathrm{g} / \mathrm{mL}$ stock solution of harpagide. IS working solution was prepared to suitable concentration of $200 \mathrm{ng} / \mathrm{mL}$. All solutions were kept at $4^{\circ} \mathrm{C}$ and were brought to room temperature before use.

Calibration standards were prepared as follows: $4 \mu \mathrm{L}$ of catalpol and harpagide 
working solution was pipetted into an Eppendorf tube and evaporated to dryness by a gentle stream of nitrogen. Then $20 \mu \mathrm{L}$ of blank rat plasma was added to create calibration curve $(20,50,100,200,500,1000,2000$ and $5000 \mathrm{ng} / \mathrm{mL}$ for catalpol and 10, 25, 50, 100, 250, 500, 1000 and $2500 \mathrm{ng} / \mathrm{mL}$ for harpagide). QC samples were prepared by the same procedure as above at concentrations of 20 , 40, 400 and $4000 \mathrm{ng} / \mathrm{mL}$ for catalpol and 10, 20, 200 and $2000 \mathrm{ng} / \mathrm{mL}$ for harpagide. $\mathrm{QC}$ samples were stored at $-80^{\circ} \mathrm{C}$ for stability evaluation.

\subsection{Sample Preparation}

An aliquot of $20 \mu \mathrm{L}$ plasma sample was transferred to an Eppendorf tube. Then $20 \mu \mathrm{L}$ of IS working solution and $100 \mu \mathrm{L}$ of acetonitrile were added. The mixtures were vortex-mixed for $2 \mathrm{~min}$ and centrifuged at 13,000 rpm for $10 \mathrm{~min}$. The supernatant was diluted 1:2 with mobile phase and then vortex-mixed for 30 s. An aliquot of $2 \mu \mathrm{L}$ of the mixture was injected into the LC-MS/MS system.

\subsection{Method Validation Procedure}

Specificity was assessed by comparing chromatograms obtained from six different batches of blank rat plasma with those obtained from corresponding standard samples spiked with catalpol, harpagide and IS, and a rat plasma sample after oral administration of ZYD. The linearity of the assay was assessed by analyzing the calibration curves using least-squares linear regression of the peak area ratios of the analytes to the IS versus the nominal concentration of the calibration standard with a weighed factor $\left(1 / \mathrm{x}^{2}\right)$. QC samples at low, medium and high concentration were analyzed on three separate occasions with six replicates at each concentration per occasion to determine the accuracy and precision. Precision was defined as the relative standard deviation (RSD \%) and accuracy as relative error (RE \%). The recoveries of catalpol and harpagide were determined at three QC levels with six replicates by comparing the peak areas from extracted samples with those in post-extracted samples spiked with the analytes at the same concentration. The matrix effect was measured at three QC levels by comparing the peak response of blank bio-sample extracts spiked with the analytes (A) with that of pure standard solution containing equivalent amounts of the compounds $(\mathrm{B})$. The ratio $(\mathrm{A} / \mathrm{B} \times 100) \%$ was used to evaluate the matrix effect. Stability studies in bio-samples were also conducted at three QC levels in several different storage conditions: at room temperature for $6 \mathrm{~h}$, at $-80^{\circ} \mathrm{C}$ for at least 10 days, after three freeze-thaw cycles, and for $8 \mathrm{~h}$ in processed samples at $10^{\circ} \mathrm{C}$ in auto-sampler tray. Each concentration was analyzed in three replicates.

\subsection{Application to a Pharmacokinetic Study}

The method was successfully applied to generate the plasma concentration versus time profile of catalpol and harpagide in rat plasma. Before the experiment, six rats were fasted overnight with free access to water. Aqueous solutions of ZYD were given to rats at a dose of $23.7 \mathrm{mg} / \mathrm{kg}$ of catalpol and $12.8 \mathrm{mg} / \mathrm{kg}$ of harpagide. Whole blood samples (about $0.1 \mathrm{~mL}$ ) were collected from the fossa 
orbitalis vein into heparinized centrifuge tubes at 10, 20, 30 and $45 \mathrm{~min}, 1,1.5,2$, $3,4,6,8,12$ and $24 \mathrm{~h}$ after oral administration of ZYD. These samples were separated for plasma by centrifugation for $5 \mathrm{~min}$ at $2200 \times \mathrm{g}$ and then stored at $-80^{\circ} \mathrm{C}$ until further analysis. The pharmacokinetic parameters of catalpol and harpagide in rats were calculated by DAS3.0 software supplied by the Pharmacological Society of China (Beijing, China). All data were expressed as means \pm standard deviation.

\section{Results}

\subsection{Optimization of LC-MS/MS Condition}

Mass spectrometric parameters were optimized in negative ionization mode with infusion and flow injection analysis. It was observed that the signal intensity of $\left[\mathrm{M}+\mathrm{HCOO}^{-}\right]^{-}$ions were much higher than $[\mathrm{M}-\mathrm{H}]^{-}$ions for catalpol and harpagide, while the signal intensity of $[\mathrm{M}-\mathrm{H}]^{-}$ion for IS was much higher. Therefore, $\left[\mathrm{M}+\mathrm{HCOO}^{-}\right]^{-}$ions for catalpol $(\mathrm{m} / \mathrm{z} 407.2)$ and harpagide $(\mathrm{m} / \mathrm{z} 409.2),[\mathrm{M}-\mathrm{H}]^{-}$ ion for IS (m/z 294.0) were chosen as precursor ions. The most abundant and stable fragment ions at m/z 199.0 for catalpol, m/z 201.0 for harpagide and $\mathrm{m} / \mathrm{z}$ 214.0 for IS were selected for the product ions. Figure 1 displays the full-scan product ion mass spectra of $\left[\mathrm{M}+\mathrm{HCOO}^{-}\right]^{-}$ions of catalpol and harpagide. In order to reduce MS contamination, a diverter valve was installed between HPLC and MS to send HPLC eluent to waste from 0 to $0.8 \mathrm{~min}$.

The analytes and IS showed a good retention on a Waters SunFireTM $\mathrm{C}_{18}$ column (100 mm $\times 2.1 \mathrm{~mm}, 3.5 \mu \mathrm{m}$; Waters, Milford, MA, USA) with a gradient elution program. In order to find the most sensitive analytical condition, several parameters were investigated including composition of mobile phase, and type (formic acid, acetic acid, or ammonium formate) and concentration of additives. Finally, acetonitrile-10 $\mathrm{mM}$ ammonium formate with gradient elution resulted in symmetrical peak shapes and high sensitivity for all the analytes.

\subsection{Sample Cleanup}

Sample preparation is the rate-limiting step in achieving high-throughput sample analysis. It is necessary to develop simple and efficient sample cleanup after devoid of matrix effects and interference from endogenous plasma components for estimation of the analytes in rat plasma. Compared with liquid-liquid extraction or solid phase extraction, protein precipitation (PPT) has some advantages, such as high extraction efficiency, environmentally lower toxicity and simple procedure. A simple and rapid one-step protein precipitation method was adopted in this study as follows: $20 \mu \mathrm{L}$ of plasma was precipitated with $100 \mu \mathrm{L}$ of acetonitrile and the supernatant was diluted 1:2 with mobile phase prior to injection.

\subsection{Method Validation}

This assay was carried out according to the guidelines for bioanalytical method 
validation as issued by the FDA Center for Drug Evaluation and Research (2001).

Selectivity and the lower limit of quantification The specificity of the method was established with blank, pooled and individual plasma samples from six different sources. The results showed no interference with catalpol, harpagide and IS, as shown in Figure 2. The limits of detection for catalpol and harpagide with the signal-to-noise over 3 were $2 \mathrm{ng} / \mathrm{mL}$ and $0.5 \mathrm{ng} / \mathrm{mL}$, respectively. The lower limits of quantification (LLOQ) for catalpol and harpagide in plasma with the signal-to-noise over 15 were 20 and $10 \mathrm{ng} / \mathrm{mL}$, respectively. At these concentrations, the intra- and inter-day CVs were less than $14.3 \%$, and the REs were within $\pm 13.2 \%$ for both catalpol and harpagide, meeting the requirements.

Linearity The linearity was evaluated on three separate occasions with two sets calibration curves per occasion. Calibration curves were best fitted by quadratic regression with $1 / \mathrm{x}^{2}$ as the weighting factor. Correlation coefficients (r) of 0.993 or higher were obtained for catalpol and harpagide, indicating that the linearity were good over the concentration ranges of 20 to $5000 \mathrm{ng} / \mathrm{mL}$ for catalpol ( $\mathrm{y}=$ $(0.02173 \pm 0.00192) \mathrm{x}+(0.00095 \pm 0.00043))$, and 10 to $2500 \mathrm{ng} / \mathrm{mL}$ for harpagide $(\mathrm{y}=(0.03127 \pm 0.00374) \mathrm{x}+(0.00149 \pm 0.00051))$. All standards met criteria of $<15 \%$ deviation from nominal concentration.

Precision and accuracy The within day assay precision were estimated by

(a) Catalpol

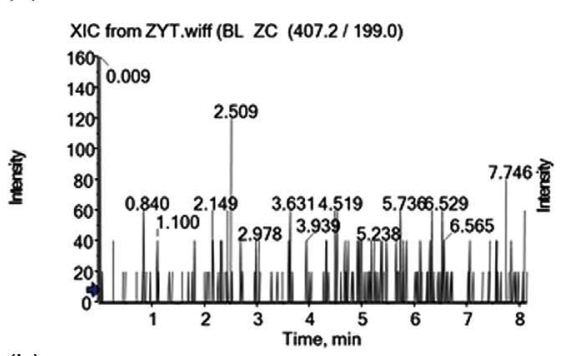

(b) XIC from ZYT.wiff (LLOQ ZC (407.2/ 199.0)

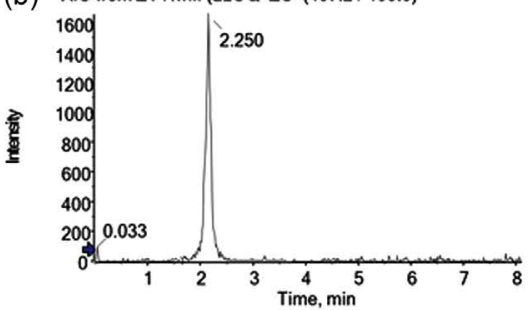

(C) XIC from ZYT.wiff (Sample ZC $(407.2 / 199.0)$

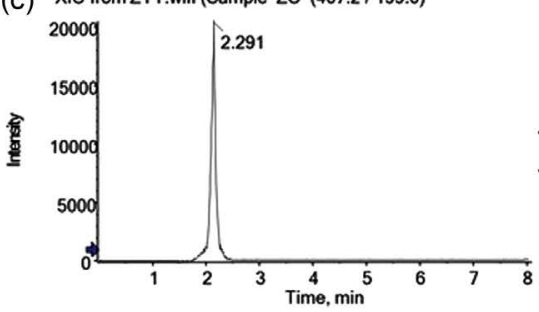

Harpagide
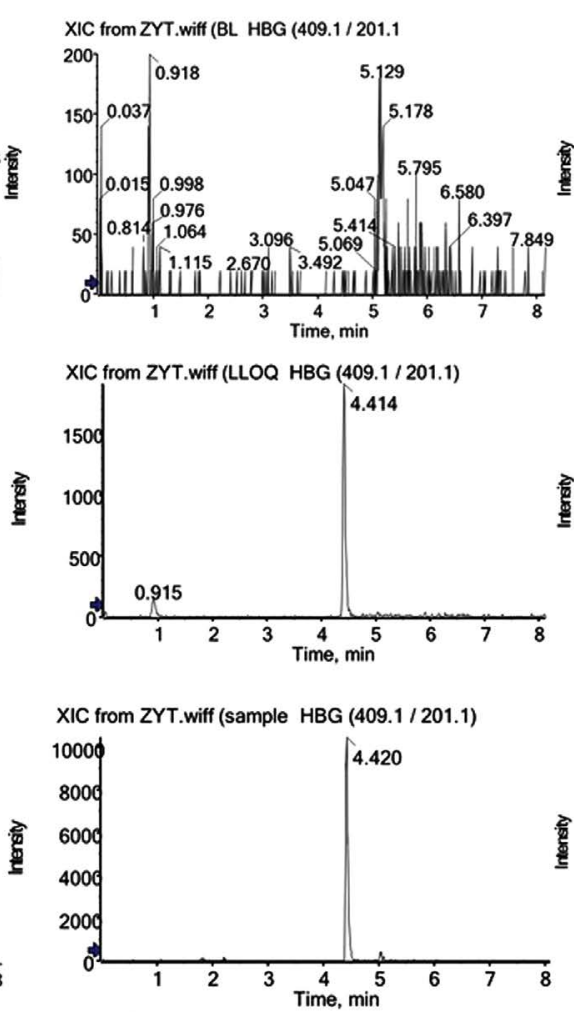

IS
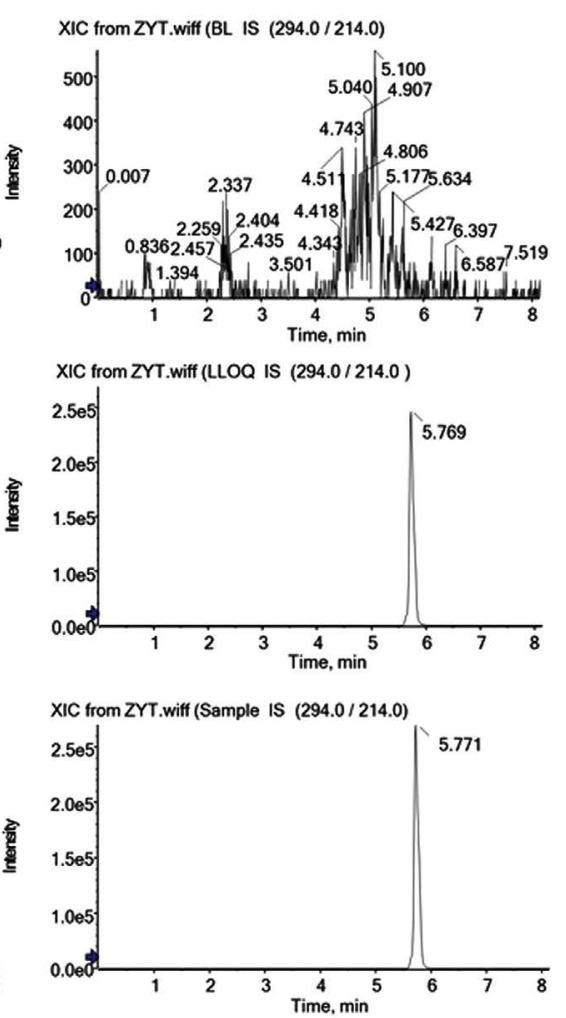

Figure 2. Representative MRM chromatograms of catalpol, harpagide and IS in rat plasma (a): a blank rat plasma; (b): a blank rat plasma spiked with catalpol (LLOQ), harpagide (LLOQ) and IS; C: a rat plasma $0.33 \mathrm{~h}$ after oral administration of ZYD. 
analyzing six replicates containing analytes of catalpol and harpagide at three different QC levels. Between day assay precision and accuracy were determined by analyzing the three level QC samples on three different runs. Table 1 shows the results obtained for the within batch and between batch precisions and accuracies of catalpol and harpagide. The precision (\% RSD) for all these analytes under investigation did not exceed $15 \%$. Accuracy (\% RE) for all analytes was in the range from $-5.9 \%$ to $6.5 \%$ at any of the concentrations studied and met the requirements of validation.

Recovery and matrix effect The recovery of catalpol and harpagide from plasma was estimated at three QC levels. Plasma samples (in six replicates) containing all analytes at QC concentration level were also spiked with internal standard. Comparing the peak areas from extracted samples with those from post-extracted blank plasma samples spiked with the pure standards at the same concentration, the ratios of the peak response were within the acceptable limit. The recoveries ranged from $79.4 \%$ to $81.2 \%$ and $78.9 \%$ to $83.1 \%$ for catalpol and harpagide, respectively. The mean recovery of the IS was $82.7 \%$ at a concentration of $200 \mathrm{ng} / \mathrm{mL}$. The results are shown in Table 1.

The matrix effect was measured at three QC levels by comparing the peak response of blank plasma extracts spiked with the analytes (A) with that of pure standard solution containing equivalent amounts of the compounds (B). The ratio $(\mathrm{A} / \mathrm{B} \times 100) \%$ was used to evaluate the matrix effect. All the ratios are listed in Table 1 and were within the range $94.3 \%-103.6 \%$ for catalpol, and $97.1 \%$ $108.2 \%$ for harpagide, indicating that no significant matrix effect was observed for catalpolorharpagide. The mean matrix effect for the IS was $100.9 \%$ at a concentration of $200 \mathrm{ng} / \mathrm{mL}$.

Stability QC samples were subjected to short-term (Room temperature for 6 h) and long-term $\left(-80^{\circ} \mathrm{C}\right.$ for 10 days) storage condition, freeze-thaw stability (three times), and autosampler stability (processed samples at $10^{\circ} \mathrm{C}$ in atuosampler for $8 \mathrm{~h}$ ). All stability studies were carried out at three concentration levels in three replicates. The results of stability experiments are summarized in

Table 1. Precisions, accuracies, recoveries and matrix effects for analysis of catalpol, harpagideand IS in rat plasma $(n=6)$.

\begin{tabular}{ccccccc}
\hline \multirow{2}{*}{ compound } & $\begin{array}{c}\text { Concentration } \\
\text { spiked }(\mathrm{ng} / \mathrm{mL})\end{array}$ & Intra-day & Inter-day & Accuracy & Recovery & Matrix effect \\
\cline { 3 - 7 } & 40 & RSD \% & RSD \% & $($ RE \% $)$ & $(\%$, mean \pm SD $)$ & $(\%$, mean \pm SD) \\
\hline \multirow{2}{*}{ catalpol } & 400 & 5 & 12.9 & 6.5 & $81.2 \pm 10.4$ & $94.3 \pm 6.9$ \\
& 4000 & 3.3 & 6.8 & -5.9 & $80.6 \pm 6.1$ & $99.8 \pm 4.8$ \\
harpagide & 20 & 8.8 & 5.2 & 2.2 & $83.1 \pm 5.7$ & $97.1 \pm 9.7$ \\
& 200 & 4.5 & 5.2 & 5.5 & $80.8 \pm 2.9$ & $108.2 \pm 3.4$ \\
IS & 2000 & 4.8 & 13.2 & 3.9 & $78.9 \pm 3.5$ & $105.5 \pm 6.5$ \\
& 200 & - & - & - & $82.7 \pm 5.4$ & $100.9 \pm 4.7$
\end{tabular}

RSD: Relative standard deviation. RE: Relative frror. SD: Standard deviation. 
Table 2. All results for the samples tested were within the acceptable criteria of $\pm 15 \%$, indicating that catalpol and harpagide were stable under the conditions described above.

\subsection{Pharmacokinetic Study}

The validated method was applied to investigate the pharmacokinetics of catalpol and harpagide in rats after intragastric administration of ZYD. Figure 3 shows the mean plasma concentrations of catalpol and harpagidevs time. The main pharmacokinetic parameters estimated by noncompartmental analysis using DAS3.0 software are listed in Table 3.

Catalpol plasma concentration reached a maximum at $0.95 \pm 0.11 \mathrm{~h}$ after administration with an average $\mathrm{C}_{\max }$ of $4.850 \pm 1.475 \mathrm{mg} / \mathrm{L}$. The average area under the curve $\left(\mathrm{AUC}_{0}-\infty\right)$ was $23.95 \pm 9.01 \mathrm{mg} \mathrm{h} / \mathrm{L}$.

Harpagide plasma concentration reached a maximum at $0.70 \pm 0.45 \mathrm{~h}$ after administration with an average $\mathrm{C}_{\max }$ of $0.371 \pm 0.098 \mathrm{mg} / \mathrm{L}$. The average $\mathrm{AUC}_{0}-\infty$ was $1.928 \pm 0.549 \mathrm{mg} \mathrm{h} / \mathrm{L}$.

Table 2. Stability of catalpol and harpagide in rat plasma $(n=3)$.

\begin{tabular}{|c|c|c|c|c|c|c|c|c|c|}
\hline \multirow[t]{2}{*}{ Compound } & \multirow{2}{*}{$\begin{array}{c}\text { Concentration } \\
\text { spiked } \\
(\mathrm{ng} / \mathrm{mL})\end{array}$} & \multicolumn{2}{|c|}{$\begin{array}{l}\text { Room } \\
\text { temperature } \\
\text { for } 6 \mathrm{~h}\end{array}$} & \multicolumn{2}{|c|}{$-80^{\circ} \mathrm{C}$ for 10 days } & \multicolumn{2}{|c|}{$\begin{array}{l}\text { Three times } \\
\text { freeze-thaw } \\
\text { cylcles }\end{array}$} & \multicolumn{2}{|c|}{$\begin{array}{c}\text { Processed samples } \\
\text { in atuosampler } \\
\text { for } 8 \mathrm{~h}\end{array}$} \\
\hline & & $\mathrm{RE} \%$ & RSD \% & RE \% & RSD \% & $\mathrm{RE} \%$ & RSD \% & $\mathrm{RE} \%$ & RSD \% \\
\hline \multirow{3}{*}{ catalpol } & 40 & 3.6 & 4.1 & -1.9 & 6.3 & 4.1 & 9.4 & 1.4 & 3.9 \\
\hline & 400 & 2.7 & 3.9 & -5.1 & 5.4 & -0.9 & 11.2 & -3.3 & 4.2 \\
\hline & 4000 & 3.2 & 7.1 & -3.8 & 5.9 & 4.9 & 3.3 & -4.1 & 13.1 \\
\hline \multirow{3}{*}{ harpagide } & 20 & 1.1 & 5.9 & 0.3 & 7.0 & 6.7 & 12.1 & -0.9 & 2.2 \\
\hline & 200 & 4.8 & 3.1 & -4.1 & 3.9 & 2.0 & 7.8 & -3.2 & 4.9 \\
\hline & 2000 & -2.7 & 4.7 & -6.3 & 9.7 & 4.7 & 2.9 & -5.9 & 7.1 \\
\hline
\end{tabular}

Table 3. The main pharmacokinetic parameters of catalpol and harpagide after oral administration of ZYD (Mean \pm SD; $n=6$ ).

\begin{tabular}{cccc}
\hline & & Catalpol & Harpagide \\
\cline { 3 - 4 } Parameters & units & $($ mean $\pm \mathrm{SD})$ & $($ mean $\pm \mathrm{SD})$ \\
\hline $\mathrm{AUC}_{(0-\mathrm{t})}$ & $\mathrm{mg} \cdot \mathrm{L}^{-1} \mathrm{~h}$ & $23.75 \pm 9.05$ & $1.666 \pm 0.693$ \\
$\mathrm{AUC}_{(0-\infty)}$ & $\mathrm{mg} \cdot \mathrm{L}^{-1} \mathrm{~h}$ & $23.95 \pm 9.01$ & $1.928 \pm 0.549$ \\
$\mathrm{t}_{\mathrm{l} / 2 \mathrm{z}}$ & $\mathrm{h}$ & $2.04 \pm 0.59$ & $5.06 \pm 2.29$ \\
$\mathrm{~T}_{\max }$ & $\mathrm{h}$ & $0.95 \pm 0.11$ & $0.70 \pm 0.45$ \\
$\mathrm{CL}_{\mathrm{z}} / \mathrm{F}$ & $\mathrm{L} \cdot \mathrm{h}^{-1} \cdot \mathrm{kg}^{-1}$ & $1.116 \pm 0.429$ & $6.978 \pm 1.511$ \\
$\mathrm{~V}_{\mathrm{z}} / \mathrm{F}$ & $\mathrm{L} \cdot \mathrm{kg}^{-1}$ & $2.99 \pm 0.29$ & $52.67 \pm 18.73$ \\
$\mathrm{C}_{\max }$ & $\mathrm{mg} \cdot \mathrm{L}^{-1}$ & $4.850 \pm 1.475$ & $0.371 \pm 0.098$ \\
\hline
\end{tabular}



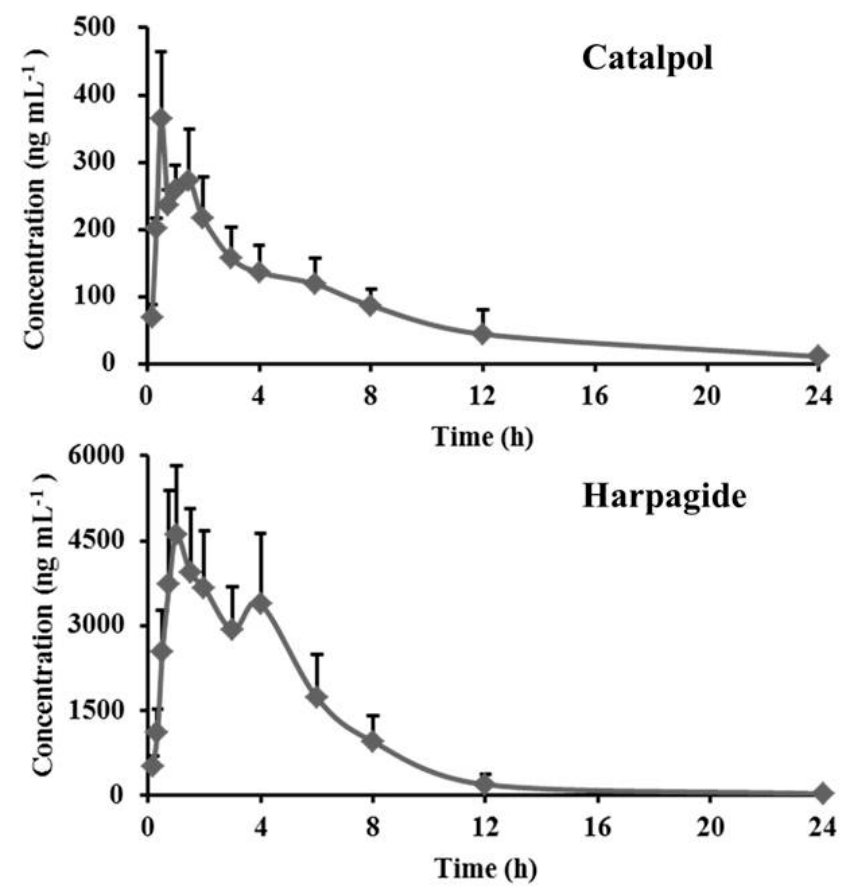

Figure 3. Mean concentration time curves of catalpol and harpagide in rat plasma after the oral administration of ZYD $(n=6)$.

\section{Discussion}

Our microsampling method was found to cause minimal stress to the rats, and a maximum of $1.3 \mathrm{~mL}$ whole blood could be collected for 13 time points over a 24-hour time period. Furthermore, after centrifugation and isolation of plasma, a small volume $(20 \mu \mathrm{L})$ was found to be sufficient for LC-MS/MS quantification. One-step protein precipitation method was adopted in this study achieving high-throughput sample analysis.

After intragastric administration of ZYD, catalpol and harpagide were rapidly absorbed and reached its peak concentrations in rats. Catalpol and harpagide have showed a double-peak phenomenon, which was usually seen in the pharmacokinetic study of herb medicine [22] [23] [24] [25]. Absorption of drugs (especially for herb medicines) from the gastrointestinal tract was a complex process and the phenomenon might be caused by variability of absorption within different regions of the gut or transformation of different compounds [23] [26]. Some iridoidglucosides were hydrolyzed by enzymes such as $\beta$-glucosidase [12] [27]. Catalpol or harpagide may be biotransformed from other components in ZYD, which should be clarified in the further studies.

\section{Conclusions}

A simple, fast and sensitive LC-MS/MS method has been developed and validated for the simultaneous quantification of catalpol and harpagide in rat plasma after oral administration of ZYD. Compared with previously published methods, this method provides major advantages in terms of less plasma volume 
$(20 \mu \mathrm{L})$, shorter run time less $(8 \mathrm{~min})$ and wider linear range (20 to $5000 \mathrm{ng} / \mathrm{mL}$ for catalpol and 10 to $2500 \mathrm{ng} / \mathrm{mL}$ for harpagide). The method was successfully applied to pharmacokinetic study of catalpol and harpagide in rats. The obtained in vivo data for catalpol and harpagide will be helpful to better understand the pharmacological effect of ZYD.

\section{Acknowledgements}

This work was supported by Yangtze Fund for Youth Teams of Science and Technology Innovation (2016cqt04). All authors have read and approved the final manuscript.

\section{References}

[1] Wu, X., Ren, C., Zhou, H., Zhang, L., Juan, C. and Yang, Y. (2014) Therapeutic Effect of Zeng Ye Decoction on Primary Sjogren's Syndrome via Upregulation of Aquaporin1 and Aquaporin5 Expression Levels. Molecular Medicine Reports, 10, 429-434. https://doi.org/10.3892/mmr.2014.2208

[2] Liu, J.Y., Zheng, C.Z., Hao, X.P., Zhang, D.J., Mao, A.W. and Yuan, P. (2016) Catalpol Ameliorates Diabetic Atherosclerosis in Diabetic Rabbits. American Journal of Translational Research, 8, 4278-4288.

[3] Shieh, J.P., Cheng, K.C., Chung, H.H., Kerh, Y.F., Yeh, C.H. and Cheng, J.T. (2011) Plasma Glucose Lowering Mechanisms of Catalpol, an Active Principle from Roots of Rehmannia glutinosa, in Streptozotocin-Induced Diabetic Rats. Journal of Agricultural and Food Chemistry, 59, 3747-3753. https://doi.org/10.1021/jf200069t

[4] Zhou, J., Xu, G., Ma, S., Li, F., Yuan, M. and Huang, K. (2015) Catalpol Ameliorates High-Fat Diet-Induced Insulin Resistance and Adipose Tissue Inflammation by Suppressing the JNK and NF-kappaB Pathways. Biochemical and Biophysical Research Communications, 467, 853-858. https://doi.org/10.1016/j.bbrc.2015.10.054

[5] Bi, J., Jiang, B., Zorn, A., Zhao, R.G., Liu, P. and An, L.J. (2013) Catalpol Inhibits LPS plus IFN-Gamma-Induced Inflammatory Response in Astrocytes Primary Cultures. Toxicology in Vitro, 27, 543-550. https://doi.org/10.1016/j.tiv.2012.09.023

[6] Fu, K., Piao, T., Wang, M., Zhang, J., Jiang, J., Wang, X. and Liu, H. (2014) Protective Effect of Catalpol on Lipopolysaccharide-Induced Acute Lung Injury in Mice. International Immunopharmacology, 23, 400-406.

https://doi.org/10.1016/j.intimp.2014.07.011

[7] Chen, Y., Zhang, Y., Xu, M., Luan, J., Piao, S., Chi, S. and Wang, H. (2017) Catalpol Alleviates Ovalbumin-Induced Asthma in Mice: Reduced Eosinophil Infiltration in the Lung. International Immunopharmacology, 43, 140-146. https://doi.org/10.1016/j.intimp.2016.12.011

[8] Yang, T., Zheng, Q., Wang, S., Fang, L., Liu, L., Zhao, H., Wang, L. and Fan, Y. (2017) Effect of Catalpol on Remyelination through Experimental Autoimmune Encephalomyelitis Acting to Promote Olig1 and Olig2 Expressions in Mice. BMC Complementary and Alternative Medicine, 17, 240. https://doi.org/10.1186/s12906-017-1642-2

[9] Xia, Z., Zhang, R., Wu, P., Xia, Z. and Hu, Y. (2012) Memory Defect Induced by Beta-Amyloid plus Glutamate Receptor Agonist Is Alleviated by Catalpol and Donepezil through Different Mechanisms. Brain Research, 1441, 27-37. https://doi.org/10.1016/j.brainres.2012.01.008 
[10] Chen, W., Li, X., Jia, L.Q., Wang, J., Zhang, L., Hou, D., Wang, J. and Ren, L. (2013) Neuroprotective Activities of Catalpol against CaMKII-Dependent Apoptosis Induced by LPS in PC12 Cells. British Journal of Clinical Pharmacology, 169, 1140-1152. https://doi.org/10.1111/bph.12200

[11] Jia, Y., Gong, N., Li, T.F., Zhu, B. and Wang, Y.X. (2015) Peptidic Exenatide and Herbal Catalpol Mediate Neuroprotection via the Hippocampal GLP-1 Receptor/Beta-Endorphin Pathway. Pharmacological Research, 102, 276-285. https://doi.org/10.1016/j.phrs.2015.10.008

[12] Zhang, L., Feng, L., Jia, Q., Xu, J., Wang, R., Wu, Y. and Li, Y. (2011) Effects of Beta-Glucosidase Hydrolyzed Products of Harpagide and Harpagoside on Cyclooxygenase-2 (COX-2) in Vitro. Bioorganic \& Medicinal Chemistry, 19, 4882-4886. https://doi.org/10.1016/j.bmc.2011.06.069

[13] Chung, H.J., Kyung Kim, W., Joo Park, H., Cho, L., Kim, M.R., Kim, M.J., Shin, J.S., Ho Lee, J., Ha, I.H. and Kook Lee, S. (2016) Anti-Osteoporotic Activity of Harpagide by Regulation of Bone Formation in Osteoblast Cell Culture and Ovariectomy-Induced Bone Loss Mouse Models. Journal of Ethnopharmacology, 179, 66-75. https://doi.org/10.1016/j.jep.2015.12.025

[14] Schopohl, P., Gruneberg, P. and Melzig, M.F. (2016) The Influence of Harpagoside and Harpagide on TNFalpha-Secretion and Cell Adhesion Molecule mRNA-Expression in IFNgamma/LPS-Stimulated THP-1 Cells. Fitoterapia, 110, 157-165. https://doi.org/10.1016/j.fitote.2016.03.005

[15] Feng, Y., Liu, Z., Peng, Y., Zhang, L., Ju, P., Bi, K. and Chen, X.H. (2013) Validated LC-MS Method for Simultaneous Quantitation of Catalpol and Harpagide in Rat Plasma: Application to a Comparative Pharmacokinetic Study in Normal and Diabetic Rats after Oral Administration of Zeng-Ye-Decoction. Biomedical Chromatography, 27, 1503-1510. https://doi.org/10.1002/bmc.2949

[16] Wang, Y., Liao, D., Qin, M. and Li, X. (2016) Simultaneous Determination of Catalpol, Aucubin, and Geniposidic Acid in Different Developmental Stages of Rehmannia glutinosa Leaves by High Performance Liquid Chromatography. Journal of Analytical Methods in Chemistry, 49, 565-589.

[17] Zhao, M., Tao, J., Qian, D., Liu, P., Shang, E.P., Jiang, S., Guo, J., Su, S.L., Duan, J.A. and Du, L. (2016) Simultaneous Determination of Loganin, Morroniside, Catalpol and Acteoside in Normal and Chronic Kidney Disease Rat Plasma by UPLC-MS for Investigating the Pharmacokinetics of Rehmannia glutinosa and Cornus officinalis Sieb Drug Pair Extract. Journal of Chromatography B Analytical Technology in the Biomedical and Life Science, 1009-1010, 122-129. https://doi.org/10.1016/j.jchromb.2015.12.020

[18] Tao, J.H., Zhao, M., Wang, D.G. Yang, C., Du, L.Y., Qiu, W.Q. and Jiang, S. (2016) Biotransformation and Metabolic Profile of Catalpol with Human Intestinal Microflora by Ultra-Performance Liquid Chromatography Coupled with Quadrupole Time-of-Flight Mass Spectrometry. Journal of Chromatography B Analytical Technology in the Biomedical and Life Science, 1009-1010, 163-169.

[19] Colas, C., Popot, M.A., Garcia, P., Bonnaire, Y. and Bouchonnet, S. (2008) Analysis of Iridoids from Harpagophytum and Eleutherosides from Eleutherococcus senticosus in Horse Urine. Biomedical Chromatography, 22, 912-917. https://doi.org/10.1002/bmc.1030

[20] Colas, C., Garcia, P., Popot, M.A., Bonnaire, Y. and Bouchonnet, S. (2008) Optimization of Solid-Phase Extraction for the Liquid Chromatography-Mass Spectrometry Analysis of Harpagoside, 8-Para-Coumaroyl Harpagide, and Harpagide in 
Equine Plasma and Urine. Journal of Chromatography Science, 46, 174-183. https://doi.org/10.1093/chromsci/46.2.174

[21] Wen, B., He, R., Li, P., Xu, P., Lu, Y., Peng, B. and Li, J. (2013) Pharmacokinetics of 8-O-Acetylharpagide and Harpagide after Oral Administration of Ajuga decumbens Thunb Extract in Rats. Journal of Ethnopharmacology, 147, 503-508. https://doi.org/10.1016/j.jep.2013.03.048

[22] Chen, L., Liu, Y., Jia, D., Yang, J., Zhao, J., Chen C., Liu, H. and Liang, X. (2016) Pharmacokinetics and Biodistribution of Aurantiamide and Aurantiamide Acetate in Rats after Oral Administration of Portulaca oleracea L. Extracts. Journal of Agricultural and Food Chemistry, 64, 3445-3455. https://doi.org/10.1021/acs.jafc.6b00470

[23] Yu, Y., Li, Q., Bi, K., Xie, P., Yang, G. and Chen, X. (2011) A Sensitive Liquid Chromatography-Mass Spectrometry Method for Simultaneous Determination of Alisol A and Alisol A 24-Acetate from Alisma orientale (Sam.) Juz. in Rat Plasma. Analytical and Bioanalytical Chemistry, 399, 1363-1369. https://doi.org/10.1007/s00216-010-4426-9

[24] Xu, Y., Li, Y., Zhang, P., Yang, B., Wu, H., Guo, X., Li, Y. and Zhang, Y. (2017) Sensitive UHPLC-MS/MS Quantitation and Pharmacokinetic Comparisons of Multiple Alkaloids from Fuzi-Beimu and Single Herb Aqueous Extracts Following Oral Delivery in Rats. Journal of Chromatography B Analytical Technology in the Biomedical and Life Science, 1058, 24-31. https://doi.org/10.1016/j.jchromb.2017.05.016

[25] Meng, X., Zhao, X., Long, Z., Yuan, Y., Zhang, H., Bi, K. and Chen, X.H. (2013) A Sensitive Liquid Chromatography-Mass Spectrometry Method for Simultaneous Determination of Three Diterpenoid Esters from Euphorbia lathyris L. in Rat Plasma. Journal of Pharmaceutical and Biomedical Analysis, 72, 299-305. https://doi.org/10.1016/j.jpba.2012.09.005

[26] Akao, T., Kawabata, K., Yanagisawa, E., Ishihara, K., Mizuhara, Y., Wakui, Y., Sakashita, Y. and Kobashi, K. (2000) Baicalin, the Predominant Flavone Glucuronide of Scutellariae Radix, Is Absorbed from the Rat Gastrointestinal Tract as the Aglycone and Restored to Its Original Form. Journal of Pharmaceutical and Biomedical Analysis, 52, 1563-1568.

[27] Pankoke, H., Buschmann, T. and Müller, C. (2013) Role of Plant $\beta$-Glucosidases in the Dual Defense System of Iridoid Glycosides and Their Hydrolyzing Enzymes in Plantagolanceolata and Plantago Major. Phytochemistry, 94, 99-107. https://doi.org/10.1016/j.phytochem.2013.04.016 